model reasonably describes the effect of support flexibility and specimen length upon ductility, as well as strength. Furthermore, a simple one-element model coupled to series as well as parallel springs will be analyzed and the disparate effects of these springs upon ductility will be elucidated.

It must be emphasized, however, that attention will be restricted to uniaxial THE ENGINEERING MECHANICS DIVISION

\section{Statistical Stability EfFects in Concrete Fallure}

By Zdenĕk P. Bažant, ' M. ASCE and Liisa Panula ${ }^{2}$

\section{Nature of Problem and Objectives}

In contrast to metals, the inelastic behavior of concrete as well as rocks is characterized by strain-softening, i.e., decline of stress at increasing strain. This property reduces the ductility of concrete structures and may cause sudden failure. Some investigators suggest that failure due to strain-softening may be a stability problem $(1,4,5,10,13,14)$, but they differ in the choice of the instability mode. In a preceding paper (1), it was proposed that the proper instability mode is the so-called strain localization-a mode in which the strain localizes into a narrow band that has a certain finite thickness, $l_{a}$, depending exclusively on material properties. Strain localization is a concept that has recently been receiving keen attention in continuum mechanics (16), mainly with regard to shear failures in soils and rock. In Ref. 1 , this concept was applied to concrete, analyzing a simple model of a uniaxially stressed specimen loaded by a spring. Stability analysis of strain localization in this specimen has yielded simple expressions for ductility (i.e., ratio of strain at failure $\epsilon_{f}$ to strain at peak stress $\epsilon_{p}$ ) as a function of length $l$ of the specimen and spring constant, $C_{s}$, of the supporting spring. The failure of statically indeterminate beams was analyzed as curvature localization in a similar way (1).

The purpose of this study is to extend the preceding work (1) by incorporating statistical microscopic nonhomogeneity of the test specimen into the analysis of strain-localization instability. A model of parallel elements of nonuniformly and randomly distributed properties will be used. This model is similar to that previously analyzed by Dougill (4), but differs by the fact that the failure of individual elements depends not only on their strain or stress, but also on the current stiffness properties of the whole system. It will be shown that the

Note.-Discussion open until March 1, 1979. To extend the closing date one month. a written request must be filed with the Editor of Technical Publications, ASCE. This paper is part of the copyrighted Journal of the Engineering Mechanics Division, Proceedings of the American Society of Civil Engineers, Vol. 104, No. EM5, October, 1978. Manuscript was submitted for review for possible publication on May 3, 1977

'Prof. of Civ. Engrg., Northwestern Univ., Evanston, Ill.

${ }^{2}$ Grad. Research Asst., Northwestern Univ., Evanston, Ill. longitudinal strain-localization models, which are justified by their simplicity and agreement of their predictions with experimental knowledge. but definitely neither give a complete picture of specimen behavior, nor describe the actual micromechanism of failure. In this respect, other, more physically based approaches (e.g., Ref. 12) will be required.

\section{Effect of Support flexibility ano Elastic Restraint upon Ductility}

Before turning attention to a system of elements, it is useful to analyze a specimen that is coupled first in series with a spring of spring constant, $C_{x}^{\prime}$, then in parallel with a spring of spring constant, $C_{p}$, and finally in series with spring of spring constant, $C_{s}$ (see Fig. 1). Let the specimen be initially in an

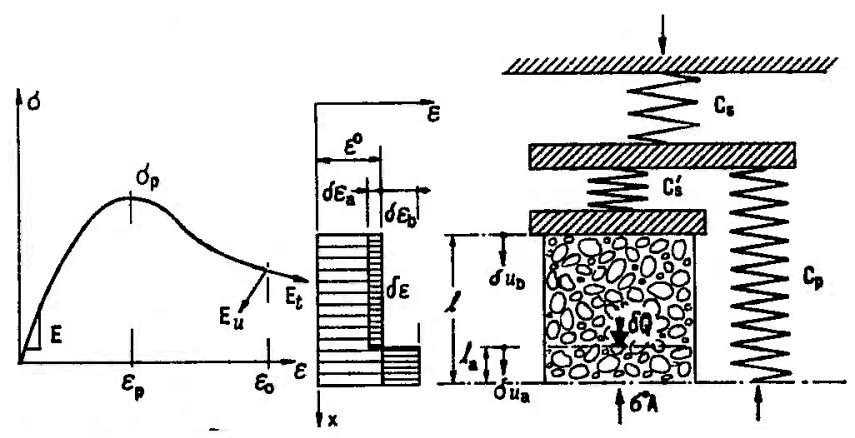

FIG. 1.-Concrete Specimen with Parallel and Series Springs, Its Stress-Strain Diagram and Straip'Localization

equilibrium state that is characterized by a uniformly distributed uniaxial stress, $\sigma$, and strain, $\epsilon$, corresponding to a point on the strain-softening branch (Fig. 1). To examine stability of this equilibrium state, assume that the strain undergoes izcrements $\delta \epsilon(x)$ of piece-wise constant distribution, by which the strain gets localized into a narrow band of thickness $2 l_{a}$ (Fig. 1). Let the displacement increments of the cross section at the boundary of the localization band and at the end of the specimen be denoted as $\delta u_{a}$ and $\delta u_{b}$, respectively. The sign of $\delta u_{a}$ and $\delta u_{b}$ will be assumed such that the localization band, $2 l_{a}$, undergoes a further increase of strain and the rest of the specimen (segments $l_{b}$ ) undergoes a strain decrease (unloading).

The simplest way to decide the question of stability is to calculate the load $\delta Q$ that must be applied at the boundary cross section of the localization band in order to produce displacement $\delta u_{a}$. The combined stiffness of springs $C_{s}$ and $C_{p}$ is $C_{s}+C_{p}$ because these springs undergo equal deformations and the reactions are added. The flexibilities of segment $l_{b}$ of the specimen and of thè spring system are $\left(l-l_{a}\right) / E_{u} A$ and $\left(1 / C_{s}^{\prime}\right)+1 /\left(C_{s}+C_{p}\right)$, respectively. 
Thus, the combined effective stiffness of the system of the three springs, together with the segment $l_{b}$, is

$$
C_{\text {eff }}=\frac{1}{\frac{l-l_{a}}{E_{u} A}+\frac{1}{C_{s}+C_{p}}+\frac{1}{C_{s}^{\prime}}}
$$

in which $E_{u}=$ unloading modulus (Fig. 1); and $A=$ cross-sectional area of the specimen. Equilibrium in the cross section at the boundary of the localization band requires that $\delta Q-E_{t} A \delta u_{a} / l_{a}-C_{\text {eff }} \delta u_{a}=0$, in which $E_{t}=$ tangent modulus, which is negative for the strain-softening branch. This may be rewritten as $\delta Q=a_{11} \delta u_{a}$, in which

$a_{11}=C_{\mathrm{efr}}+\frac{E_{t} A}{l_{a}}$

The work that is done by force $\delta Q$ to produce displacement $\delta u_{a}$ is

$\Delta W=\frac{1}{2} \delta Q \delta u_{a}=\frac{1}{2} a_{11}\left(\delta u_{a}\right)$

If $\Delta W$ is positive, then no deformation increment can occur if no work is done, which indicates stability (Drucker's postulate). If $\Delta W$ is negative and there are no frictional forces present, as in our problem, the system is unstable. (However, this is not necessarily true when frictional forces exist, although the case $\Delta W=0$, representing neutral equilibrium, might still be regarded as the stability limit for practical purposes.) Therefore, the stability condition is $\Delta W=(1 / 2) a_{11}\left(\delta u_{a}\right)^{2}>0$ or $a_{11}>0$. Substituting here Eqs. 2 and 1 , the stability condition may be brought to the form

$$
-\frac{E_{t}}{E_{u}}<\frac{1}{\frac{l}{l_{a}}-1+\frac{E_{u} A}{C_{s} l_{a}}\left(\frac{1}{1+\frac{C_{p}}{C_{s}}}+\frac{C_{s}}{C_{s}^{\prime}}\right)}
$$

This condition reduces to Eq. 8 from Ref. 1 when springs $C_{p}$ and $C_{s}^{\prime}$ are absent, i.e., $C_{s}^{\prime}=\infty, C_{p}=0$.

If the localization band could be made arbitrarily narrow, i.e., $l_{a} / l \rightarrow 0$, the tangent modulus, $E_{r}$, could not assume any negative value without violating the stability condition, Eq. 4. However, it is known from experiments that a negative slope (a strain-softening branch) does exist. To explain this experimental fact with the present model, it may be assumed that $l_{a}$ cannot be smaller than a certain value (1). This is logical because the material is not a homogeneous continuum. Since the stress and deformation of segment $l_{a}$ is described by macroscopically uniform stress and strain, thickness $l_{a}$ obviously cannot be considered to be less than the size of inhomogeneities, such as the maximum aggregate size, $d$, or else the foregoing equations would become invalid. Thus, $l_{a}=n d$, in which $n$ is a material constant, probably between 1 and 20 , and perhaps $n=2$.

To show some consequences of the stability condition (Eq. 4), a specific, empirical stress-strain diagram of concrete has been considered, using the well-known approximate relation (15)

$$
\sigma=\frac{m}{m-1+\left(\frac{\epsilon}{\epsilon_{p}}\right)^{m}} \frac{\sigma_{p}}{\epsilon_{p}} .
$$

in which $\sigma_{p}=$ peak stress; $\epsilon_{p}=$ corresponding strain; and $m=$ constant, usually

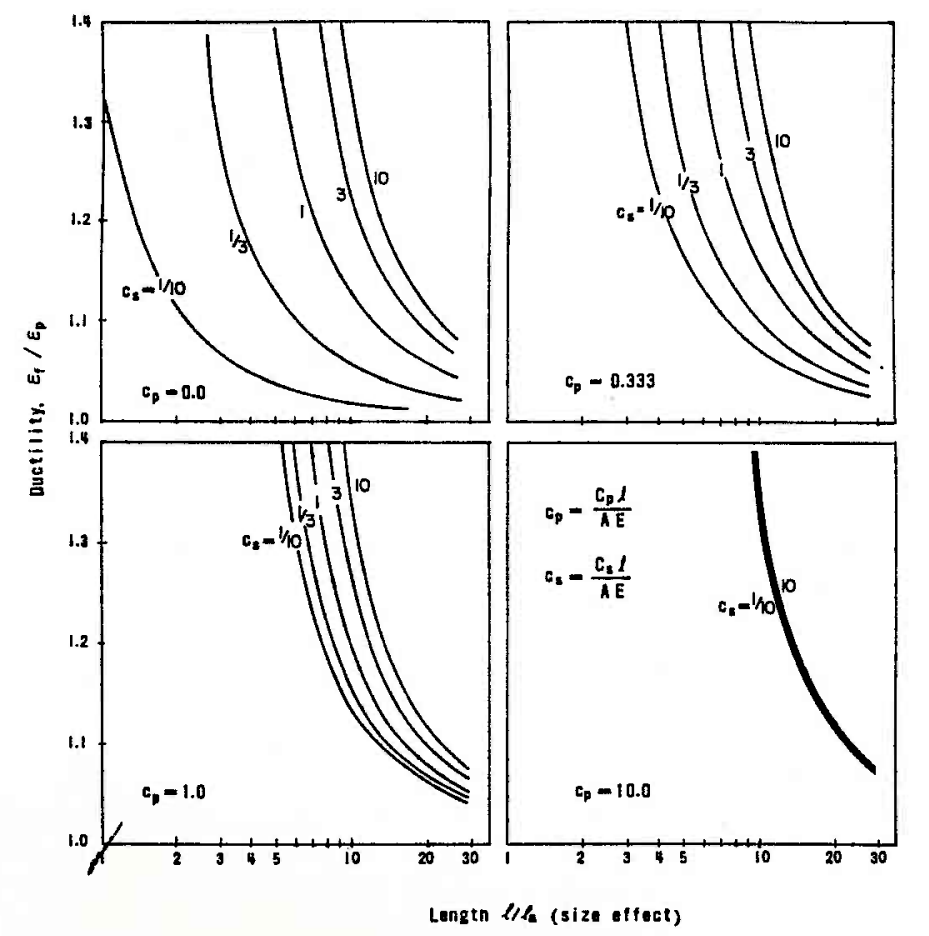

FIG. 2.-Ductility Dependence on Length and Stiffness of Series and Parallel Springs $\left(C_{s}, C_{p}\right)$, According to Eq. 6

between 2 and 3 . The tangent modulus may be obtained as $E_{\mathrm{r}}=d \sigma / d \epsilon$, which yields

$-\frac{E_{l}}{E}=\frac{(m-1)^{2}\left[\left(\frac{\epsilon}{\epsilon_{p}}\right)^{m}-1\right]}{\left[\left(\frac{\epsilon}{\epsilon_{p}}\right)^{m}-1+m\right]^{2}}$

in which $E=\left(\sigma_{p} / \epsilon_{p}\right) m /(m-1)=$ initial elastic modulus; and $m=0.0004$ $\left(\sigma_{p} / \mathrm{psi}\right)+1.0$ (where $\left.\mathrm{psi}=6.89 \mathrm{kN} / \mathrm{m}^{2}\right)$. The points $(\epsilon, \sigma)$ where the stability condition (Eq. 4) is violated first represent the failure points $\left(\epsilon_{f}, \sigma_{f}\right)$ and the 
ratio of $\epsilon_{f}$ (at failure) to $\epsilon_{p}$ (at peak stress) may be called ductility.

Substituting the right-hand side of Eq. 4 for $-E_{t} / E$ in Eq. 6, and solving the resulting equation, the diagrams in Fig. 2 (for $C_{s}^{\prime}=0$ ) have been obtained. It is seen that ductility decreases as the size of the specimen increases, and for very long specimens there is no ductility $\left(\epsilon_{f} \approx \epsilon_{p}\right)$. Making the supports more flexible by adding a series spring, $C_{5}$, destabilizes the system and decreases ductility; the more flexible is spring $C_{s}$, the larger is the ductility decrease. By contrast, providing elastic restraint by attaching a parallel spring, $C_{p}$, has a strong stabilizing effect; ductility becomes larger with a larger stiffness of the parallel spring.

Although the condition in Eq. 4 has not been rigorously formulated, the foregoing conclusions have, in essence, been intuitively recognized by experimentalists; see, e.g., the work of Evans and Marathe (6) who coupled stiff parallel elastic rods to their test specimens to enable observation of the strain-softening branch in a testing machine that would otherwise be insufficiently
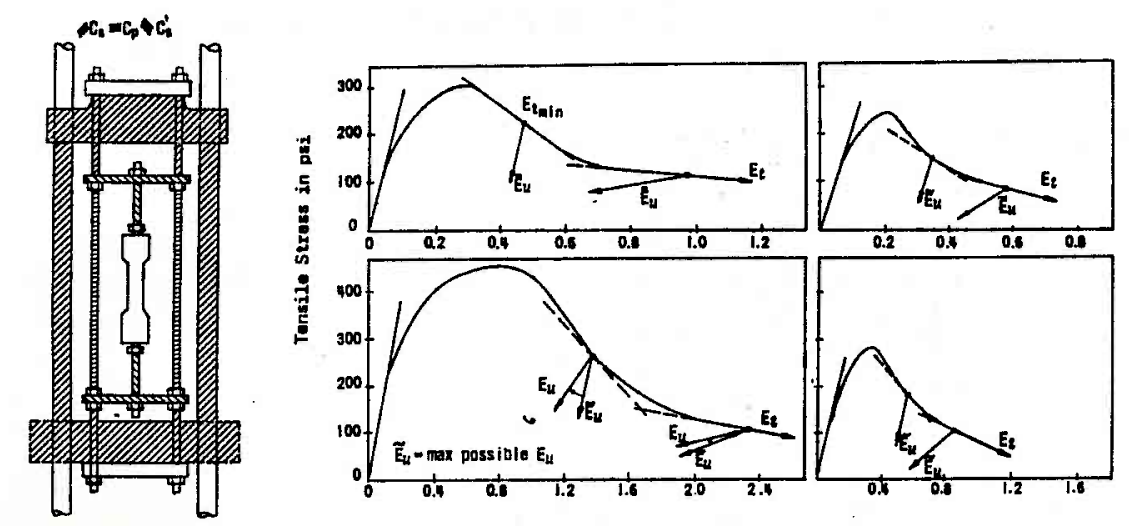

Tensale Sersean $\times 10^{3}$

FIG. 3.-Arrangement of Evans and Marathe's Tensile Tests with Slopes at Failure According to Eq. 6

stiff for this purpose. Using Eq. 4 , the spring constant, $C_{p}$, needed to stabilize the specimen could have been determined precisely.

Eq. 4 has been used to analyze tensile ductility as observed by Evans and Marathe (6). From the drawing of the testing machine (Fig. 3) given in Ref. 6 , it was estimated that $C_{s}=15 \times 10^{6} \mathrm{lb} / \mathrm{in} .(2,627 \mathrm{MN} / \mathrm{m}), C_{p}=11 \times$ $10^{6} \mathrm{lb} /$ in. $(1,926 \mathrm{MN} / \mathrm{m})$, and $C_{s}^{\prime}=4 \times 10^{6} \mathrm{lb} / \mathrm{in}$. $(700 \mathrm{MN} / \mathrm{m})$. The width of the strain-softening band was assumed as $l_{a}=2 \mathrm{in.}(50.8 \mathrm{~mm})$.

The tensile tests of Evans and Marathe show that failure occurs at a point beyond the inflection point of the descending branch, where $-E$, is less than maximum. According to Eq. 4, it would be impossible for the specimen to fail at one $-E_{t} / E_{u}$ value and not to fail at a higher $-E_{t} / E_{u}$ value. At constant $E_{u}$, failure cannot occur at points of positive curvature of softening branch, beyond an inflection point. Therefore, in order to explain Evans and Marathe's tests, it must be assumed that the unloading modulus, $E_{u}$, is decreasing as

the strain increases. The largest possible value of $E_{u}$ at the observed failure point may be calculated from Eq. 4. The unloading slopes obtained in this manner for Evans and Marathe's tests from the foregoing crude estimates of $C_{p}, C_{x}$, and $C_{s}^{\prime}$ are plotted in Fig. 3. These slopes are not unrealistic, since it is well known that at very large strain in the softening regime the unloading modulus indeed decreases and tends to approach the secant modulus. This phenomenon is due to microfracturing.

However, apart from a decrease in $E_{u}$, failure at points of positive curvature of strain-softening branch could be also explained by transverse inhomogeneity of the specimen, which is analyzed in the sequel, and by multiaxial stress effects. Probably all phenomena are at play at the same time. Nevertheless, the present simple stability model for ductility gives realistic and, above all, simple results.

\section{Parallel Multi-Element System of Nonuniformly Distaibuted Properties}

On the basis of instability caused by strain softening, the stiffness of the testing machine has been shown to affect ductility. However. no effect on

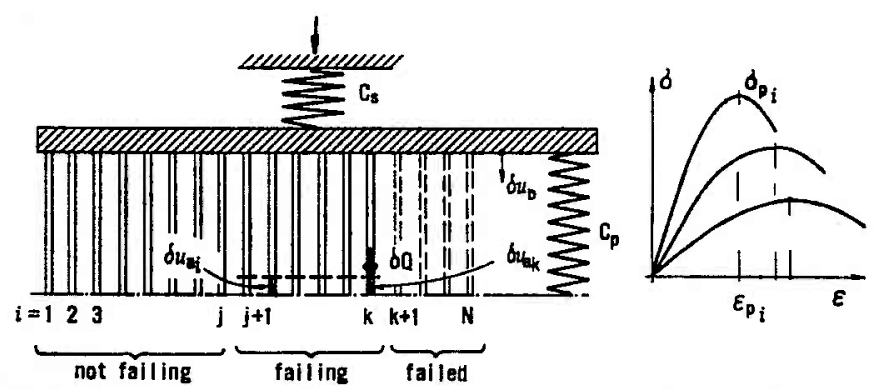

FIG. 4.-Parallel Multi-Element System of Nonuniform Properties, with Stress-Strain Curves of Elements of Various Strengths<smiles>C1C2CC1C2</smiles>

strength is predicted. Yet, it is known from experiments $(7,8)$ that strength, too, is affected by stiffness of the testing machine. This effect can be modeled in fracture mechanics (7) by means of the energy release rate, but such an explanation is hardly justified in case of concrete specimens that are not much larger than the aggregate size [6-in. $\times 12-\mathrm{in} .(152-\mathrm{mm} \times 305-\mathrm{mm})$ cylinders] because on this scale concrete does not follow Griffith-type crack propagation criteria. It will now be shown that uniaxial unstable strain localization due to strain softening is also capable of giving a simple model for the effect of machine stiffness upon strength, provided that macroscopic nonhomogeneity of the test specimen in the transverse direction is taken into account.

Consider now a system of $N$ independent parallel elements whose properties are nonuniformly distributed over the system (Fig. 4). Let each of the elements, numbered as $i=1,2, \ldots, N$, be of equal length $2 l$, while the cross-sectional areas, $A_{i}$, and material properties vary from element to element. The system of elements is loaded through symmetric springs of spring constant $C_{s}$. In addition, a spring of spring constant, $C_{p}$, is coupled in parallel with the system.

. The load, $P$, is transferred onto the elements by perfectly rigid platens (Fig. 
4) which ensure that the change of length of all elements is the same. A uniform uniaxial stress, $\sigma_{i}$, is assumed to exist within each element. The elements are numbered as $i=1,2, \ldots, N$ in the order of increasing strength. As the ends of springs are being compressed, individual elements gradually fail, beginning with the element of highest strength, $N$, because it has lowest $\epsilon_{p}$ as is typical of concretes of various strengths. Let $k$ denote the number of elements that have not yet failed at the current loading stage; i.e., elements $i=k+1$, $k+2, \ldots, N$ are those that have already failed and carry no stress; $\sigma_{i}=$ 0 for $i>k$ (Fig. 4).

To examine failure as a phenomenon of instability, all kinematically admissible variations of displacements should be analyzed. However, for the sake of simplicitly (as in Ref. 1), attention will be restricted to those displacement variations for which all cross sections of each element remain plane, the stress state remains uniaxial, and instability within any of the elements can occur only by longitudinal strain localization, in which one region, of thickness $2 l_{a}$ (Fig. $1)$, undergoes a uniform infinitesimal strain increment, $\delta \epsilon_{a j}$, while the adjacent regions, of length $l_{b}$, undergo uniform infinitesimal strain decrement $\delta \epsilon_{b_{i}}$. It is assumed that $\delta \epsilon_{a_{i}}$ represents loading $\left(\delta\left|\epsilon_{a_{i}}\right| \geq 0\right.$ ), possibly at decreasing stress, and decrement, $\delta \epsilon_{h_{i}}$ represents unloading $\left(\delta\left|\epsilon_{b_{l}}\right| \leq 0\right)$, always at decreasing stress. The sign of strain as well as stress is chosen to be negative for compression. Similarly, as before, thickness, $l_{a}$ of the strain-localization band must be an empirical material property; $l_{a}=n d$, in which $n$ is an empirical coefficient. Assuming the maximum aggregate sizes, $d$, to be the same in all elements, $l_{a}$ must be also the same, and so must be $l_{b}$.

During a given loading step, a certain number of elements may fail by unstable strain localization; they will be numbered as $i=j+1, \ldots, k$ and their number is $k-j(0 \leq j<k)$; while $i=1,2, \ldots, j$ are the elements that are not failing during the given loading step and have a uniform strain increment, $\delta \epsilon_{i}$, all over the element (Fig. 4).

The displacement increments of the rigid platens will be denoted as $\delta u_{b}$, and the displacement increments of the cross sections at the boundary of the strain-localization band will be denoted as $\delta u_{a_{t}}$ (Fig. 4). If some of the elements fail, springs tend to release energy and transfer it into the concrete. Thus, the rigid platens must move in such a direction that strain in concrete increases. The tangent modulus for loading (increasing strain, $\delta|\epsilon|>0$ ) will be denoted as $E_{t_{i},}$ and the unloading modulus as $E_{u_{i}}$. On the softening branch, $E_{t_{i}}<0$.

The simplest approach to stability analysis is to enforce a displacement, $\delta u_{a_{k}}$, of the cross section that bounds the localization band, and calculate the force, $\delta Q$, needed to produce $\delta u_{a_{k}}$ while all the rest of the system remains in equilibrium. (This approach is predicated upon the assumption that $\delta u_{a_{k}} \neq 0$ in the instability mode.) Equilibrium of forces acting at the rigid platens requires that

$$
\sum_{i=1}^{j} A_{i} E_{t_{i}} \frac{\delta u_{b}}{l}+\sum_{i=j+1}^{k} A_{i} E_{u_{i}} \frac{\delta u_{b}-\delta u_{a_{i}}}{l_{b}}+C_{s} \delta u_{b}+C_{p} \delta u_{b}=0 \ldots \ldots
$$

in which $\delta u_{b} / l=\delta \epsilon_{b}=$ strain increments in nonfailing elements; and $\left(\delta u_{b}\right.$ $\left.\delta u_{a_{i}}\right) / l_{b}=\delta \epsilon_{b_{i}}=$ strain increments in failing elements. Forces acting on the cross sections at the boundaries of the strain-localization bands in elements $i=j+1, \ldots, k$ are in equilibrium if $\delta \sigma_{b_{i}}-\delta \sigma_{a_{i}}=0$ in elements $i=j+$

$1, \ldots, k-1$, and $A_{k} \delta \sigma_{b_{k}}-A_{k} \delta \sigma_{a_{k}}+\delta Q=0$ in element $i=k$. This yields

$A_{i} E_{u_{i}} \frac{\delta u_{b}-\delta u_{a_{i}}}{l_{b}}-A_{i} E_{t_{i}} \frac{\delta u_{a_{i}}}{l_{a}}=0$ for $i=j+1, \ldots, k-1 \ldots \ldots$

and $A_{k} E_{u_{k}} \frac{\delta u_{b}-\delta u_{a_{k}}}{l_{b}}-A_{k} E_{t_{k}} \frac{\delta u_{a_{k}}}{l_{a}}+\delta Q=0$ for $i=k \ldots \ldots$

Eqs. 7-9 represent a system of $(k-m+1)$ equations for unknowns $\delta u_{b}$, $\delta u_{a_{j+1}}, \ldots, \delta u_{a_{k-1}}$, and $\delta Q$, the displacement increment, $\delta u_{a_{k}}$, being prescribed. To solve $\delta Q$ from these equations, $\delta u_{a_{i}}$ may be expressed from Eq. 8 as

$\delta u_{a_{i}}=\frac{\delta u_{b}}{1+\frac{E_{t_{i}} l_{b}}{E_{u_{i}} l_{a}}}$ for $i=j+1, \ldots, k-1 \ldots \ldots \ldots$

Substitution of this expression into Eq. 7 provides

$\delta u_{b}\left(\frac{1}{l} \sum_{i=1}^{j} A_{i} E_{t_{i}}+\sum_{i=j+1}^{k-1} \frac{A_{i}}{\frac{l_{b}}{E_{u_{i}}}+\frac{l_{a}}{E_{t_{i}}}}+\frac{A_{k} E_{u_{k}}}{l_{b}}+C_{s}+C_{p}\right)=\frac{A_{k} E_{u_{k}}}{l_{b}} \delta u_{a_{k}}$

Calculating $\delta Q$ from Eq. 9 , one has $\delta Q=A_{k}\left(E_{t_{k}} / l_{a}+E_{u_{k}} / l_{b}\right) \delta u_{a_{k}}-A_{k}\left(E_{u_{k}} / l_{b}\right)$ $\delta u_{b}$, and, expressing $\delta u_{b}$ from the preceding equation, one finally obtains $\delta Q$ $=a_{k k} \delta u_{a_{k}}$ with

$a_{k k}=A_{k}\left(\frac{E_{i_{k}}}{l_{a}}+\frac{E_{u_{k}}}{l_{b}}\right)$

$-\frac{\left(\frac{A_{k} E_{u_{k}}}{l_{b}}\right)^{2}}{\frac{1}{l} \sum_{i=1}^{j} A_{i} E_{l} /+\sum_{i=j+1}^{k-1} \frac{A_{i}}{\frac{l_{b}}{E_{u_{i}}}+\frac{l_{a}}{E_{l_{i}}}}+\frac{A_{k} E_{u_{k}}}{l_{b}}+C_{s}+C_{p}} \ldots \ldots \ldots \ldots$

The value of $\delta u_{b}$ can then be solved from Eq. 9:

$\delta u_{b}=\left(1+\frac{E_{u_{k}} l_{b}}{E_{u_{k}} l_{a}}-\frac{l_{b}}{A_{k} E_{u_{k}}} a_{k k}\right) \delta u_{a_{k}} \ldots \ldots \ldots \ldots$

and $\delta u_{a_{i}}$ can be evaluated from Eq. 10.

To examine stability, one may again consider the work

$\Delta W=\frac{1}{2} \delta Q \delta u_{a_{k}}=\frac{1}{2} a_{k k}\left(\delta u_{a_{k}}\right)^{2} \ldots \ldots \ldots \ldots \ldots \ldots$

which must be done to produce displacement $\delta u_{a_{k}}$. The stability condition is $\Delta W=(1 / 2) a_{k k}\left(\delta u_{a_{k}}\right)^{2}>0$ or $a_{k k}>0$.

If $\delta Q=0$ or $a_{k k}=0$, the system is in neutral equilibrium and the initial state is a critical state. It must be checked, however, whether the signs of 
$\delta u_{b}$ and $\delta u_{b}-\delta u_{a}$ agree with the assumed moduli. The modulus for regions $l_{a}$ of the failing elements $i=j+1, \ldots, k$ was assumed in Eq. 7 to be the unloading modulus, $E_{u_{i}}$. It may now be checked that for $a_{k k}=0$ and $E_{t_{k}}<$ 0 , Eq. 13 indicates that $\delta u_{b} / \delta u_{c_{k}}<1$, which confirms that unloading indeed takes place in regions $l_{a}$. Furthermore, from Eq. 10 it follows that $\delta u_{k} / \delta u_{a_{i}}$ $<1$ for all failing elements, because $E_{t_{i}}<0$ for these elements. (This also shows that instability cannot occur in elements whose stresses are not on the strain-softening branch.) Finally, if the downward slope of the strain-softening branch is not unreasonably large, i.e., if $-E_{t_{i}}<E_{u_{i}} l_{a} / l_{b}$, then Eq. 13, with $a_{k k}=0$, indicates that $\delta u_{b} / \delta u_{a_{k}}>0$, and this means that the use of modulus $E_{t}$ in Eq. 7 for the nonfailing elements was correct.

The special case of the condition $a_{k k}>0$ (Eq. 12) for $N=k=1$ and $C_{p}$ $=0$ is identical to Eq. 17 of Ref. 1 , concerned with a single failing element, and the same special case for $C_{p} \neq 0$ is identical to Eq. 4 for the one-element model (Fig. 1) with $C_{s}^{\prime}=0$.

\section{Ductility of Large Nonhomogeneous System of Parallel Elements}

Eq. 12 may now be used to analyze ductility of the model. Individual elements of the model exhibit different peak stress $\sigma_{p_{i}}$. Let $\phi\left(\sigma_{p}\right)$ be the probability density distribution of the amount of concrete of varying peak stress $\sigma_{p}$ within the system. Assuming that the peak stresses of individual elements vary discretely as $\sigma_{p_{1}}, \sigma_{p_{2}}=\sigma_{p_{1}}-\Delta \sigma_{p}, \ldots, \sigma_{p_{N}}=\sigma_{p_{1}}-(N-1) \Delta \sigma_{p}$, then the total cross-sectional area of all elements of peak stress $\sigma_{p}$ within the interval $\sigma_{p_{i}}-(1 / 2) \Delta \sigma_{p} \leq$ $\sigma_{p}<\sigma_{p_{i}}+(1 / 2) \Delta \sigma_{p}$ is

$A_{i}=A \Delta \sigma_{p} \phi\left(\sigma_{p_{l}}\right) ;$ with $A=\sum_{i=1}^{N} A_{i} \ldots \ldots . \ldots \ldots$

in which $A=$ combined cross-sectional area of all elements.

For numerical studies, the approximate stress-strain diagram given by Eq. 5 has been assumed again. Constant $m$ in Eq. 5 , usually between 2 and 3, determines the roundness of the peak and the maximum downward slope of the $\sigma-\epsilon$ curve. For higher $m$ values, the peak is sharper and the maximum downward slope is higher (relative to $E$ ). This is reflected in the empirical relation $m=0.0004 f_{c}^{\prime}+1(15)$ that has been used herein. For stability analysis, the location of peak stress, $\dot{\epsilon}_{p}$, and the shape of the $\sigma-\epsilon$ curve immediately after the peak, are most important. It is, therefore, more appropriate to set up a direct empirical expression for $\epsilon_{p}$ and determine $m$ on the basis of $\epsilon_{p}$. From an analysis of numerous experimental data $(9,11)$ it has been found that $\epsilon_{p}=0.0023\left(4,500 \mathrm{psi} / f_{c}^{\prime}\right)^{q}\left(\mathrm{psi}=6.89 \mathrm{kN} / \mathrm{m}^{2}\right)$ for $f_{c}^{\prime} \geq 3,000 \mathrm{psi}(20,670$ $\mathrm{kN} / \mathrm{m}^{2}$ ) in which $q$ is a constant between 0.4 and 1.0 for most types of standard-weight concretes. This expression reflects the fact that weaker concrete reaches the peak stress at higher strain (provided that $f_{c}^{\prime} \geq 3,000$ psi or 20,670 $\mathrm{kN} / \mathrm{m}^{2}$ ).

The effective unloading modulus, $E_{u}$, of the whole specimen decreases along the strain-softening branch, due to fracturing of microelements. The individual elements of our model may be regarded as such microelements, and so the value of unloading modulus $E_{u}$ for individual elements that have not yet failed may be considered to be equal to its initial value, which may be taken as E.

Having determined the stress-strain relations and the cross-sectional areas of individual elements in the model, it is now possible to calculate the strain, $\epsilon_{u}$, at which the system of parallel elements becomes unstable. For this purpose, a simple computer program has been written. Using the specified $\sigma_{p_{i}}$ values, the program finds $A_{i}, \epsilon_{p}, E_{i}=E_{u}$, and $m_{i}$ for all elements $i=1,2, \ldots, k$. Then, the program increments the value of strain $\epsilon$ in nonfailed elements proceeding in small steps $\Delta \epsilon$. For each step, $\Delta \epsilon$, the calculation procedure is as follows:

1. Using given $\sigma_{p i}$ values for elements $i=1,2, \ldots, N$, calculate $E_{t_{i}}$ (Eq. 6) and $\epsilon_{p_{i}}$ for all elements $i=1,2, \ldots, k$.

2. Find all elements $(i=j, j+1, \ldots, k)$ that are in the strain-softening regime (i.e., $\epsilon>\epsilon_{p_{i}}$ ). Begin calculation with this value of $j$, which corresponds to the assumption that all softening elements fail during the increment, $\Delta \epsilon$.

3. Evaluate $a_{k k}$ from Eq. 16 and check stability condition, $a_{k k}>0$.

4. If the stability condition is violated, go to step 5 . If it is not violated, increase $j$ by one $(j \leftarrow j+1)$, which means that fewer elements are assumed to fail during the current $\Delta \epsilon$ increment, and repeat item 3 . If for some $r$ the stability condition is violated, proceed directly to step 5. If all $j$ values up to $k-1$ are exhausted without detecting instability, no element is failing during the current increment $\Delta \epsilon$ and one has $j=k$.

5. Set $k \leftarrow j$ and calculate the mean stress, $\sigma$, at the end of increment $\Delta \epsilon$, i.e., $\sigma=\Sigma_{i} A_{i} \sigma_{i} / \Sigma_{i} A_{i}, i=1, \ldots k$, in which $\sigma_{i}$ is given by Eq. 5 for $f_{c}^{\prime}$ $=f_{c_{i}}^{\prime}, j=j_{i}$, and $\epsilon_{p}=\epsilon_{p_{i}}$.

6. Set $\epsilon \leftarrow \epsilon+\Delta \epsilon$, return to 1, and begin calculations for the next increment, $\Delta \epsilon$.

The calculations just outlined have been run for normal (Gaussian) distribution of peak stress $\sigma_{p}$, i.e.

$\phi\left(\sigma_{p}\right)=\frac{1}{s \sqrt{2 \pi}} \exp -\frac{\left(\sigma_{p}-\bar{\sigma}_{p}\right)^{2}}{2 s^{2}} \ldots \ldots \ldots \ldots$

in which $\bar{\sigma}_{p}=$ mean peak stress; and $s=$ standard deviation of $\sigma_{p}$. (Note that ACI strength $f_{c}^{\prime}$ is defined to be less than $\bar{\sigma}_{p}$.) Various values of $\bar{\sigma}_{p}, s$, spring constant $C_{s}$, length $l / l_{a}$, and of coefficient $q$ (indicating the decrease of $\epsilon_{p}$ with increasing $\sigma_{p}$ ) have been considered in the analysis. All calculations were made for a system of 34 elements; the interval $\bar{\sigma}_{p}-3 s \leq \sigma_{p} \leq \bar{\sigma}_{p}+$ $3 s$ has been subdivided in 33 equal subintervals $\Delta \sigma_{p}$; the strain increment was $\Delta \epsilon=25 \times 10^{-6}$.

The typical numerical results are plotted in Figs. 5-7. While for the case of a single element (Fig. 1) with constant unloading modulus $E_{\text {u }}$ an inflection point cannot exist on the descending branch because failure cannot occur at points of smaller $\left|E_{t}\right|$ than some previous $\left|E_{t}\right|$, for the parallel system, Fig. 5 shows that the descending branch of the diagram of mean stress versus strain does exhibit an inflection point followed by a long "tail" of progressively smaller slope and positive curvature. This is despite the fact that the unloading modulus, 
of each element (unlike the overall $E_{u}$ considered in Fig. 3 ) has been considered equal to the initial modulus, $E$. The existence of the inflection point in the descending branch for uniaxial localization model is thus seen to be a consequence of nonhomogeneity, which causes that the individual elements in the parallel system fail by instability gradually, provided that $s$ is sufficiently large. The average unloading modulus of the whole system is $\left\langle E_{u}\right\rangle=E \Sigma_{i}\left(A_{i} / A\right)$, in which $i=1,2, \ldots, k$, and it is clear the $\left\langle E_{u}\right\rangle$ decreases as $k$ becomes smaller than $N$; it is this value that corresponds to $E_{u}$ for the single element model in Fig. 1 .

The gradual element failure and the decline of $\left\langle E_{u}\right\rangle$ may also be represented by Dougill's model of parallel linearly elastic elements failing at a given, statistically distributed stress (4), but the dependence of failures on the flexibility of support and the length of specimen is not reflected in the simpler Dougill's model. Thus, the present model is an extension of Dougill's approach.

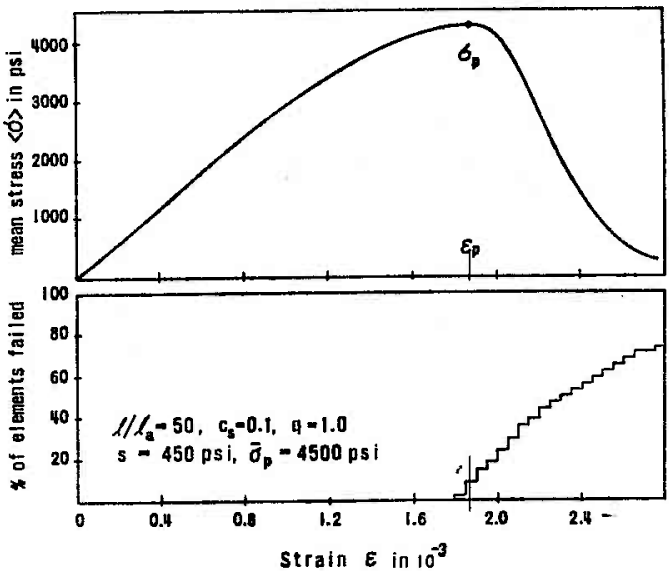

FIG. 5.-Typical Stress-Strain Diagram for Multi-Element System in Fig. 4 with Gradual Failure of Elements

The present model yields a very long tail of the descending branch, which makes it difficult to define failure and ductility precisely. Failure can no longer be defined as the point of zero stress, because the uniaxial localization would give unrealistically large ductility. It has been somewhat arbitrarily assumed that specimen disintegrates (in a triaxial manner) when $\langle\sigma\rangle$ drops below 0.25 $\langle\sigma\rangle_{p}$, and the corresponding strain has been considered to be the failure strain, $\epsilon_{f}$, for the entire system. Such values of $\langle\sigma\rangle$ at failure approximately agree with the failure points implied by the measured stress-strain diagrams of Evans and Marathe (6).

The typical results for ductility, indicating the effect of length and support flexibility upon ductility, are plotted in Fig. 6. These diagrams are seen to be very similar to those obtained for a single element (Fig. 2). Therefore, the single-element model seems to be sufficient as far as ductility is concerned (provided that the unloading modulus is reduced with increasing strain). This is not so, however, for strength.

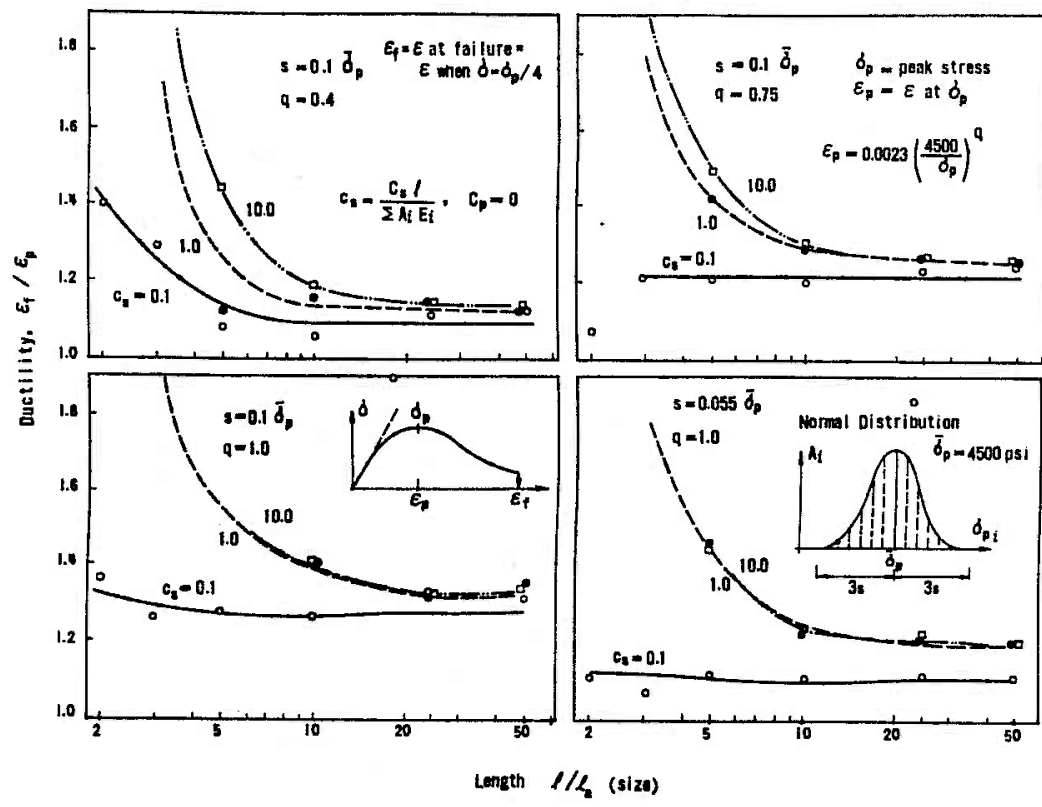

FIG. 6.-Ductility of Parallel Multi-Element System

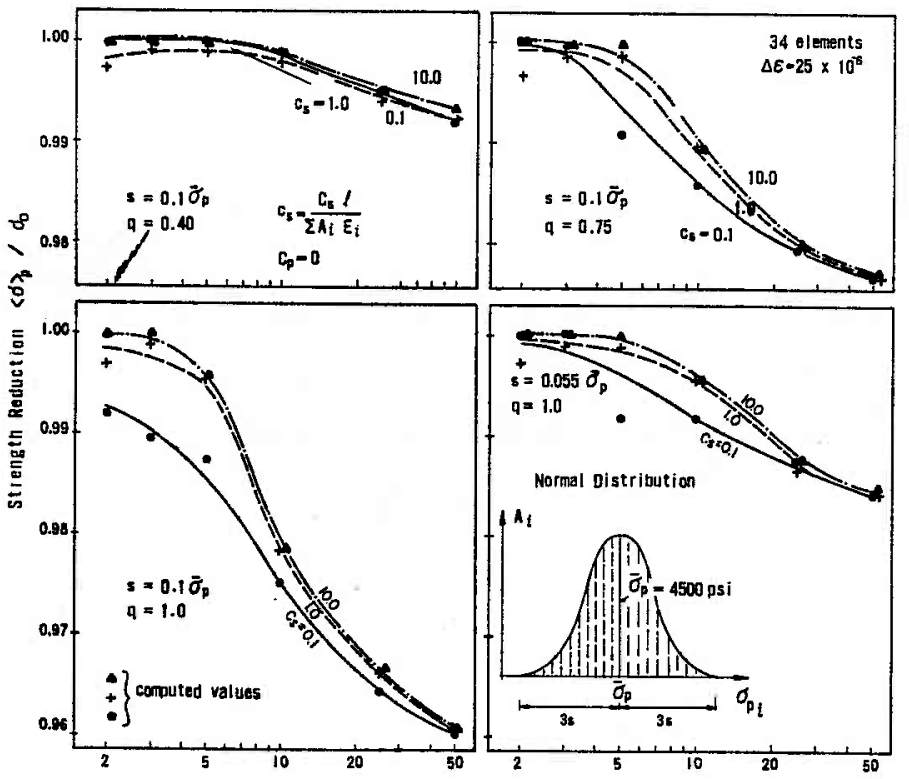

Length $1 / 10$ (siza)

FIG. 7.-Reduction of Strength of Parallel Multi-Element System due to Length and Support Floxibility 
Fig. 7 shows that an increase of length of the specimen (system of parallel elements) can reduce the peak $\langle\sigma\rangle_{p}$ of the mean stress $\langle\sigma\rangle$ (i.e., strength) appreciably (by about $4 \%, s=0.1 \bar{\sigma}_{p}$ ). Flexibility of support $C_{s}$ can cause a similar reduction of strength. These reductions are due to the fact that the strongest (not the weakest!) elements reach their individual peak stresses and suffer localization instability before the peak $\langle\sigma\rangle_{p}$ is attained by the mean stress $\langle\sigma\rangle$. This can happen only if the strain, $\epsilon_{p}$, at peak stress $\sigma_{p}$ decreases with decreasing $\sigma_{p}$, which is known to be true for concrete of strength higher than 3,000 psi $\left(20,670 \mathrm{kN} / \mathrm{m}^{2}\right)$. Because the number of the elements that become unstable before reaching $\langle\sigma\rangle_{p}$ is higher for longer specimens and for more flexible support, the strength reduction becomes stronger. That such reductions of strength indeed exist is confirmed by experiments $(7,8)$.

The standard deviation, $s$, is an important factor. The value of $0.1 \bar{\sigma}_{p}$ considered in the calculations would be high when the overall strengths of various standard test cylinders are compared. However, for the variations of the strength of microelements within a standard cylinder, the value $s=0.1 \bar{\sigma}_{p}$ would no doubt be too low. Certainly, in one test reported (8), in which a strength reduction as high as $35 \%$ was caused by placing a spring under the test specimen, it would be necessary to consider $s$ to be much higher than $0.1 \bar{\sigma}_{p}$ in order to obtain a quantitative agreement.

\section{System of Parallel Elements of Random Properties}

The reduction of strength demonstrated in the previous section is caused by instability of several strongest (and stiffest) elements in the system, which reach the strain-softening stage before the total stress attains its peak. Hidden in the analysis was a tacit assumption that the system is so wide (i.e., number $N$ of elements is so high) that the areas of the elements, $A_{i}$, can be determined from their statistical distribution. However, if the system is not sufficiently wide (i.e., if $N$ is not large), then the peak stresses, $\bar{\sigma}_{p_{i}}$, and areas, $A_{i}$, are random numbers of given statistical distribution. Since in the parallel element model the strongest elements fail first (because they are stiffest), the distribution of the areas of the strongest elements has a major effect. A rigorous way to treat it would be to develop extreme value statistics, analogous to that used to predict failure of a cable consisting of many wires. However, this approach would be rather complicated when combined with the stability conditions. Therefore, it was decided to use random (Monte Carlo) simulation by computer.

In the preceding section, the frequency of concrete elements of peak stress within a chosen interval, $\sigma_{p_{t}}-(1 / 2) \Delta \sigma_{p} \leq \sigma_{p_{l}}<\sigma_{p}+(1 / 2) \Delta \sigma_{p}$, was modeled by area $A_{i}$, associated with peak stress $\sigma_{p_{l}}$ (Eqs. 15 and 16). In Monte Carlo simulation, however, the peak stress values are not chosen, but represent individual random events; so, each element is considered to have a unit area, $A_{i}=1$, and the previous role of $A_{i}$ is now replaced by the number of elements falling within a given interval of peak stress $\Delta \sigma_{p}$. A set of $N$ random numbers $\sigma_{p_{1}}(i=1,2, \ldots N)$ of normal distribution, characterized by prescribed mean $\bar{\sigma}_{p}$ and standard deviation $s$, have been generated by computer. For this set of $\sigma_{p}$ values (with $A_{1} \equiv 1$ ), the mean stress-strain curve of $(\sigma)$ versus $\epsilon$ was calculated step by step as described in the preceding section, and the obtained peak value of $\langle\sigma\rangle_{p}$ of mean stress $\langle\sigma\rangle$ was plotted as a point in Fig. 8. The calculation has been run for $N_{s}$ different randomly generated sets of $N$ values of $\sigma_{p_{i}}$, and the obtained peak point was plotted in Fig. 8 for each of these sets; $N_{s}=10$ was used for all cases, except that $N_{s}=30$ was used for the largest $N$.

To simulate the statistical size effect of width upon localization instability. the preceding calculation for $N_{s}$ different sets of $\sigma_{p_{i}}$ values was made for various values of $N(N=2,4,8,16$ and 32). The results are all plotted in Fig. 8 As expected, in a greater number $N$ of elements there is a greater likelihood of obtaining a stiffest (and strongest) element, and so the number of elements that have reached their peak stress and failed is higher and appears to approach

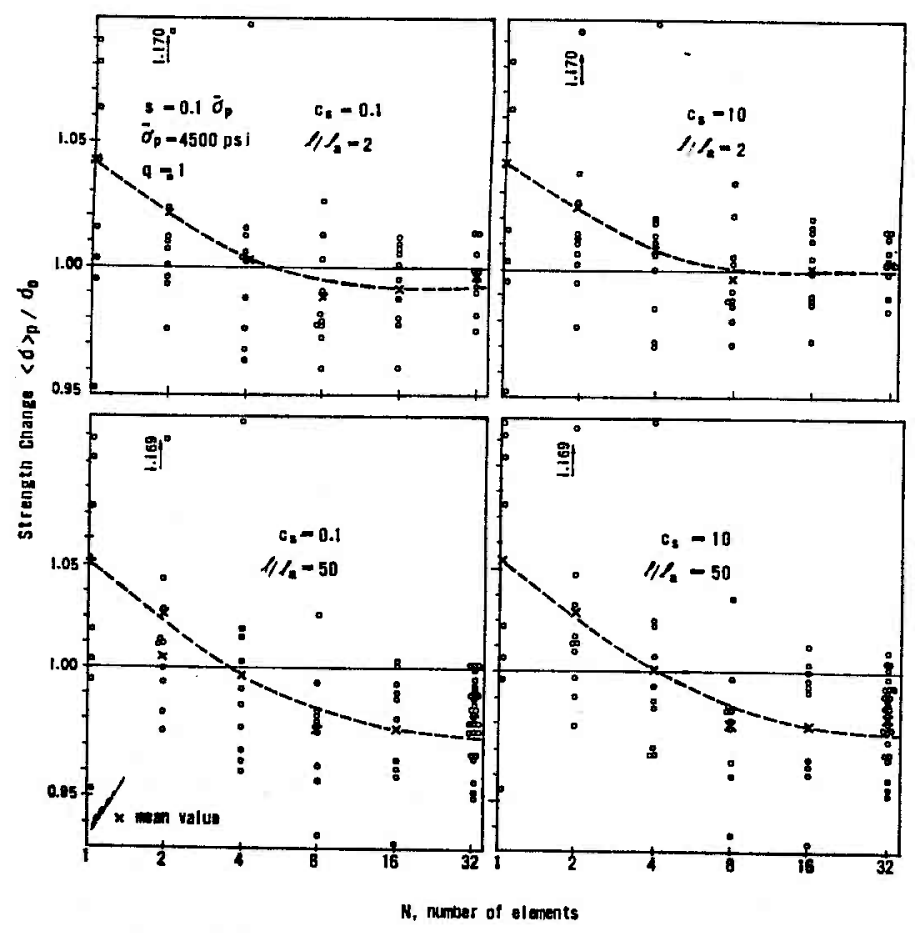

FIG. 8.-Change of Strength of Parallel Multi-Element System as Function of Number of Elements (Width), Support Flexibility, and Length

an asymptotic value as $N$ becomes very large. This phenomenon represents the statistical size effect of width upon localization instabilities.

The reduction of peak stress is due exclusively to elements of highest strength, which aiso have highest stiffness; they pass beyond their peak stress and fail by localization instability before the combined mean stress-strain curve peaks. The largest among the $\epsilon_{p_{i}}$ values corresponding to the generated $\sigma_{p_{i}}$ values becomes smaller as the number of random trials, $N$, is increasing, and so the percentage of elements that fail by localization instability before the mean stress-strain curve peaks is increasing, causing the peak of this curve to become lower as $N$ is increased. This statistical size effect contrasts with the classical 
weakest-link chain model-a series rather than a parallel system in which the weakest rather than the stiffest and strongest elements initiate failure.

To sum up, according to uniaxial localization models, one can distinguish two different nonconventional types of size effect in the strength of nonhomogeneous materials: (a) A size effect of width that is statistical in nature and is modeled by extreme value statistics (failure of stiffest elements); and (b) a size effect of length that is caused by instability due to strain localization in long specimens.

In real, three-dimensional specimens, such a clear distinction, however, does not exist. The effect of support flexibility $C_{s}$ is similar to effect (b), and the addition of elastic restraint $C_{p}$ counteracts effect (b).

If the test specimen is very short $\left(l \rightarrow l_{a}\right)$, and the supports are stiff $\left(C_{s}\right.$ $\rightarrow \infty$, the strength reduction due to instability is negligible and the statistical size effect of width is the only one in action. In this case, the strength $\langle\sigma\rangle_{p}$ increases as the width is decreased, which is shown by curve a in Fig. 9. If the test specimen is very wide (which corresponds to a system of many elements in Fig. 4), the statistical size effect is negligible and the size effect

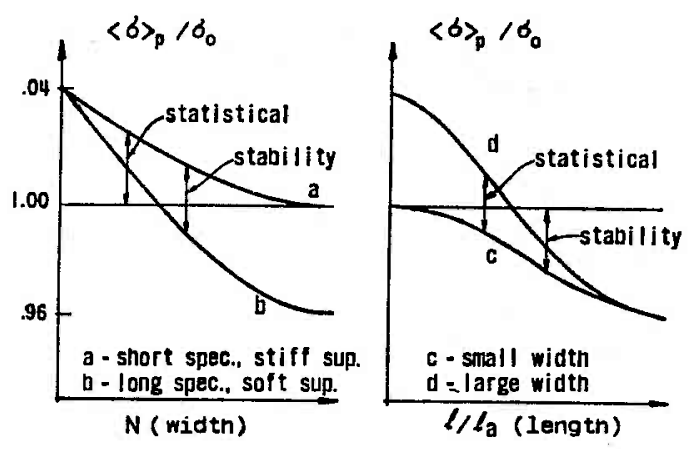

FIG. 9.-Comparison of Strength Change Due to Statistical and Stability Effects

of instability due to length or support flexibility is the only one present. This case is depicted by curve $c$ in Fig. 9. When the length is not small (large $l / l_{a}$ ) or supports are flexible (small $C$ ), and the width is not large (small $N$ ), both size effects are operative simultaneously, and the combined size effect is given by curves $b$ and $d$ in Fig. 9, based on the simulation results from Fig. 8. It is seen from Fig. 8 that both types of size effect are about equally significant, one reducing the strength as from $1.0-0.96$, the other one increasing the strength as from 1.0-1.04, the combined effect being a variation of strength as from 0.96-1.04. This is not a large effect; however, a greater variation would be obtained if a broader variation of strength of individual elements were assumed. This would be appropriate if the whole model represented the standard size cylinder, because the elements of this specimen, corresponding to the elements of the model, are rather small and have widely scattered properties.

Apart from the statistical effects analyzed, there exists, of course, the well-known classical statistical size effect on strength $(2,18)$, which is described by the "weakest link" model of a chain under tension. This effect is known to be quite significant in tensile failure of concrete but is probably of little significance in the compression failure, because the mechanics of this failure is not realistically modeled by the failure of a chain.

As far as ductility is concerned, the results of the Monte-Carlo simulations are not shown because they have been very similar for all $N$ to the results for a wide system (large $N$ ) (Fig. 6). This is obviously due to the fact that ductility, unlike strength, depends not only on the strongest elements but on all elements in the system.

\section{Conclusions}

1. Unstable strain localization in strain-softening concrete is capable of explaining the dependence of ductility, as well as strength, upon the length and the width of specimen, the flexibility of support, the parallel elastic restraint, and the degree of nonhomogeneity of concrete.

2. The fact that a parallel elastic restraint has a stabilizing effect while support flexibility destabilizes concrete specimens is quantitatively described, in a rather simple manner. by the present model (Eq. 4). The thickness of the strain-softening band, and both the tangent modulus and the unloading modulus, must be empirically known in order to be able to predict the instability.

3 . The strength reduction due to unstable strain localization can be modeled by considering a system of parallel elements of nonuniformly distributed properties reflecting macroscopic nonhomogeneity of the specimen. In contrast to the weakest link model for a chain, the reduction of strength is here due to the strongest elements, which are stiffest, and are therefore first to fail by unstable strain localization, before the total load on the system attains its peak.

4. The existence of an inflection point and a prolonged tail on the descending branch, as well as the decrease of the unloading modulus, can be explained by nonhomogeneity of the parallel element model; thus the individual elements do not suffer stability failures at the same strain. In this model, the shape of the descending branch and the location of the inflection point depends on the machine stiffness, as well as the parallel elastic restraint.

5. There exists a nonclassical statistical effect in strength, i.e., the statistical stability effect, which is due to the fact that in a wider specimen (having more parallel elements) the likelihood of encountering stiffer elements of smaller strain at peak stress is higher, causing more elements to become unstable before the cotal load attains its peak. The statistical stability effect is always intertwined with the effects of length, support flexibility and parallel elastic restraint, and depends on the standard deviation of peak stress values within the parallel system.

6. The statistical stability effect in a parallel system can model the reduction of strength due to an increase in the support flexibility or in the length of specimen.

\section{ACKNOWLedgment}

Support under National Science Foundation Grants ENG 75-14848-A01 and ENG 77-06767 is gratefully acknowledged. 


\section{Appendix 1.-References}

1. Bažant, Z. P., "Instability, Ductility, and Size-Effect in Strain-Softening Concrete," Journal of the Engineering Mechanics Division, ASCE, Vol. 102, No. EM2, Proc. Paper 12042, Apr., 1976, pp. 331-344.

2. Bolotin, V. V., Statistical Methods in Structural Mechanics, Holden-Day, San Francisco, Calif., 1969 (translated from Russian).

3. Creus, G. J., discussion of "Instability, Ductility, and Size Effect in Strain-Softening Concrete" by Zdenèk P. Bažant, Journal of the Engineering Mechanics Division, Concrete, by Zdenék P. Bazant, Paurn 12830, Apr., 1977, pp. 357-358.

4. Dougill, J. W., "A Mathematical Model for Progressive Failure of Cement Paste and Mortar," Magazine of Concrete Research, Vol. 19, 1967, pp. 135-142; see also Vol. 23, 1971, pp. 5-9.

5. Dougill, J. W., "Modes of Instability of Concrete Panels Exposed to High Temperatures," Magazine of Concrete Research, Vol. 24, 1972, pp. 71-76.

6. Evans, R. H., and Marathe, M. S., "Microcracking and Stress-Strain Curves for Concrete in Tension," Materials and Structures, Paris, France, Vol. 1, 1968, pp. 61-64.

7. Glucklich, J., and Cohen, L. J., "Size as a Factor in the Brittle Ductile Transition and the Strength of Some Materials," International Journal of Fracture Mechanics, Dec., 1967, pp. 278-289.

8. Glucklich, J., and Cohen, L. J., "Strain-Energy and Size Effects in a Brittle Material," Materials Research and Standards, American Society for Testing and Materials, Vol. 8, Oct., 1970, pp. 17-22.

9. Hognestad, E., Hanson, N. W., and McHenry, D., "Concrete Stress Distribution in Ultimate Strength Design," Journal of the American Concrete Institute, Proceedings, Vol. 52,1955 , pp. $455-477$.

10. Jurina, L., and Meier, G., discussion of "Instability, Ductility, and Size Effect in Strain-Softening Concrete," by Zdeněk P. Bažant, Journal of the Engineering Mechanics Strain-Softening Concrete, by Z EM4, Proc. Paper 13093, Aug., 1977, pp. 775-777.

11. Kupfer, H. B., "Das Verhalten des Betons unter mehrachsiger Kurzzeitbelastung unter besonderer Berücksichtigung der zweiachsigen Beanspruchung," Deutscher Ausschuss für Stáhlbeton, Heft 229, W. Ernst \& Sohn, West Berlin, East Germany, 1972.

12. McClintock, F. A., Mayson, X., The Effect of Voids on Material Deformation, S Cowin and M. M. Carroll, eds., American Society of Mechanical Engineers Publication, 1976.

13. Maier, G. “On Structural Instability Due to Strain-Softening," Proceedings, InternaMaier, G., "On Stranich tional Union of Theoretical and Applied Mechanics Symposium on Instability

14. Maier, G. Zavelani, A., and Dotreppe, J. C., "Equilibrium Branching due to Flexural Softening," Journal of the Engineering Mechanics Division, ASCE, Vol. 99, No. EM4, Proc. Paper 9898, Aug., 1973, pp. 897-901.

15. Popovics, S., "A Numerical Approach to the Complete Stress-Strain Curve of Concrete," Cement and Concrete Research, Vol. 3, 1973, pp. 583-599.

16. Rice I "The Localization of Plastic Deformation," Preprints, 14th Congress of the International Union for Theoretical and Applied Mechanics, Delft, Netherlands, 1976, pp. 207-220.

17. Sabnis, G. M. and Aroni, S "Size Effects in Material Systems--the State of the Art," Structure, Solid Mechanics, and Engineering Design, Proceedings, Southampton 1969 C. E. Materials Conference, M. Te'eni, ed., Wiley-Interscience, London, England, 1971, pp. 131-142.

18. Volkov, S. D., Statistical Strength Theary, Gordon and Breach, New York, N.Y., 1966 (translated from Russian).

\section{Appendix II.-Notation}

The following symbols are used in this paper:

\section{$a_{i j}=$ stiffness coefficients;}

spring constants of parallel spring, series springs, and effective spring constant;

$E, E_{t}, E_{u}=$ initial elastic modulus, tangent modulus, and unloading modulus;

$l, l_{a}, l_{b}=$ length of specimen and its segments (Fig. 1):

$s=$ standard deviation of $\sigma_{p}$

$u_{a}, u_{a_{i}}, u_{b}=$ axial displacements of cross sections (Figs. 1 and 4);

$\Delta W=$ second-order work at small load increments $\delta \phi$

$\epsilon, \sigma=$ strain and stress (uniaxial);

$\epsilon_{p}, \sigma_{p}=$ strain and stress at peak point (Fig. 1); and

$\bar{\sigma}_{p}=$ mean value of $\sigma_{p}$.

\section{STATISTICAL STABILITY AND CONCRETE FAILURE}

KEY WORDS: Brittle failure; Compression tests; Concrete tests; Ductility; Homogeneity; Materials stability; Size perception; Statistical analysis; Stiffness; Strain; Strength; Stress strain diagrams; Tensile testers

ABSTRACT: Using a uniaxial localization model, it is shown that parallel elastic restraint increases ductility, while an increase in support flexibility or length of specimen reduces ductility. Statistical macroscopic nonhomogeneity of the specimen is modeled by a system of uniaxial parallel elements of random properties following the normal distribution. The stability analysis and Monte Carlo simulations explain that in such a system an increase of length or support flexibility reduces not only ductility but also the strength of the system. The effect on strength depends on the number of elements (width of specimen), which represents a new non-classical statistical size effect, and on the standard deviation of peak stress values within the parallel system. Existence of an inflection point and the prolonged tail on the descending branch is explained by the nonhomogeneity of the specimen, and the shape of the descending branch, along with the location of the inflection point, is obtained as a
machine stiffness, parallel elastic restraint, and specimen length and width. REFERENCE: Bazant, Zdenek P., and Panula, Liisa, "Statistical Stability Effects in No. EMS, Proc. Paper 14074, October, 1978, pp. 1195-1212 